\title{
Ultrasensitive SERS immunoassay based on diatom biosilica for detection of interleukins in blood plasma
}

\author{
Agnieszka Kamińska $^{1}$ - Myroslav Sprynskyy ${ }^{2}$ - Katarzyna Winkler ${ }^{1}$. \\ Tomasz Szymborski ${ }^{1}$
}

Received: 26 May 2017 /Revised: 7 July 2017 / Accepted: 2 August 2017 / Published online: 29 August 2017

(C) The Author(s) 2017. This article is an open access publication

\begin{abstract}
An ultrasensitive surface-enhanced Raman scattering (SERS) immunoassay based on diatom biosilica with integrated gold nanoparticles (AuNPs) for the detection of interleukin 8 (IL-8) in blood plasma has been developed. The SERS sensing originates from unique features of the diatom frustules, which are capable of enhancing the localized surface-plasmon resonance of metal nanostructures. The SERS immune tags ware fabricated by functionalizing 70 $\mathrm{nm}$ Au nanoparticles with DTNB (i.e., 5,5'-dithiobis(2nitrobenzoic acid)), which acted as a Raman reporter molecule, as well as the specific antibodies. These DTNB-labeled immune-AuNPs can form a sandwich structure with IL-8 antigens (infection marker) and the antibodies immobilized on the biosilica material. Our method showed an improved IL-8 detection limit in comparison to standard ELISA methods. The current detection limit for IL-8 using a conventional ELISA test is about $15.6 \mathrm{pg} \mathrm{mL}^{-1}$. The lower detection limit for IL-8 in blood plasma was estimated to be $6.2 \mathrm{pg} \mathrm{mL}^{-1}$. To the best of our knowledge, this is the first report on the recognition of IL-8 in human samples using a SERS-based method. This method clearly possesses high sensitivity to clinically relevant interleukin concentrations in body fluids. The
\end{abstract}

Electronic supplementary material The online version of this article (https://doi.org/10.1007/s00216-017-0566-5) contains supplementary material, which is available to authorized users.

Agnieszka Kamińska

akamin@ichf.edu.pl

1 Institute of Physical Chemistry, Polish Academy of Sciences, Kasprzaka 44/52, 01-224 Warsaw, Poland

2 Department of Environmental Chemistry and Bioanalytics, Faculty of Chemistry, Nicolaus Copernicus University, 7 Gagarina Str, 87-100 Toruń, Poland average relative standard deviation of this method is less than $8 \%$, which is sufficient for analytical analysis and comparable to those of classical ELISA methods. This SERS immunoassay also exhibits high biological specificity for the detection of IL-8 antigens. The established SERS immunoassay offers a valuable platform for the ultrasensitive and highly specific detection of immune biomarkers in a clinical setting for medical diagnostics.

Keywords Surface-enhanced Raman spectroscopy (SERS) . Interleukin $8 \cdot$ Diatom biosilica $\cdot$ Immunoassay

\section{Introduction}

Interleukins are secreted proteins that are members of the cytokine family of immune system molecules that regulate immune cell activity. Interleukins are produced by immune system cells such as lymphocytes, macrophages, and monocytes [1]. They modulate inflammation and immunity by regulating the growth, mobility, and differentiation of lymphoid and other cells [2]. The analysis and quantitation of interleukins in body fluids is important as it allows us to broaden our understanding of their immunological functions. Cytokine levels in body fluids can provide information useful for disease diagnosis and staging, prognostication, and thus the selection of an appropriate disease therapy [3]. Several analytical procedures for quantifying interleukins in body fluids and tissue culture supernatants have been developed. Enzyme-linked immunosorbent assays (ELISA) are the most popular methods of quantitating secreted cytokines due to their high specificities and sensitivities $[4,5]$. Intracellular staining, the ribonuclease protection assay (RPA) [6], the polymerase chain reaction (PCR) [7], and cytometric assays [8] have also been used in recent decades. However, each of these techniques has at least 
one significant limitation. For instance, problems with cytokine assays, including a lack of accuracy, have been reported; a number of factors have been shown to affect the validity and quality of measurements obtained with such assays [9-12].

Therefore, there is a need to develop a more sensitive, selective, stable, and durable method for analyzing these biomarkers. Recent advances in nanotechnology and instrumentation development have permitted the development of a highly sensitive and chemically specific technique for biomolecular system recognition that uses surface-enhanced Raman spectroscopy (SERS) [13]. The phenomenon of SERS can be explained as a combination of an electromagnetic mechanism (EM) and a chemical mechanism related to charge transfer between a substrate and an adsorbed molecule [14]. Theoretically, the electromagnetic enhancement can reach factors of $10^{3}$ to $10^{11}$, whilst chemical enhancement factors of up to $10^{3}$ have been calculated $[15,16]$. This huge enhancement in Raman scattering-even single molecules can be observed - ensures that Raman spectroscopy is very effective for ultrasensitive bioanalysis [17]. Another interesting characteristic of SERS is the linear dependence of the SERS intensity on the power of the incident light, despite the nonlinear signal enhancement that is achieved with this technique. Thus, SERS could potentially be applied for the quantitative measurement of analytes with ultrahigh sensitivity.

SERS biosensing has been used to detect various biological samples and diseases, including various cancers [18], Alzheimer's disease [19], and Parkinson's disease [20]. The most notable recent advances in SERS include the application of this technique to immunosensing. SERS-based immunoassays have attracted significant research interest due to their (i) high detection sensitivities and selectivities [21], (ii) reduced susceptibility to photobleaching [21], and (iii) narrow spectral bandwidth, allowing multiplex analysis [22].

Wang et al. [23, 24] demonstrated the detection of the interleukins IL-6 and IL-8 from buffer solution. Typically, SERS immunoassays are realized on flat glass, on a noble metal ( $\mathrm{Au}, \mathrm{Ag}$, or alloys of them) surface with nanoscale roughness [25], or on photonic crystals [26]. Unfortunately, silver - which gives the best signal enhancement factors - undergoes oxidation, so when a silver surface is used, SERS must be carried out before the surface oxidizes. Photonic crystals, despite their excellent properties, are expensive to develop as techniques requiring high-tech equipment (i.e., PVD sputtering, photolithography, focused ion beam, and others) are needed.

In the work reported in the present paper, we investigated a more accessible approach based on natural photonic crystals (materials) such as diatom biosilica. Diatoms are photosynthesizing algae that possess a siliceous skeleton called a frustule [27] comprising complex hierarchical micro- to nanosized structures under natural conditions. We employed diatom silica frustules produced by Pseudostaurosira trainorii as an inexpensive and easy to prepare and modify functional material to use in a novel SERS immunoassay.

Obtaining three-dimensional (3D) structures of inorganic materials is one of the main challenges involved in the development of nanotechnology. The shape and the pattern of a frustule are unique to the particular diatomic species that produced it [28]. Thus, highly individual 3D silica structures can be obtained from single-celled diatoms without the need to use complex and expensive nanofabrication methods. Diatom frustules exhibit unpredictable optical properties due to their quasi-ordered pore patterns, such as diffraction-driven selffocusing [29] and gas-sensitive photoluminescence emission [30]. Researchers have investigated whether the unusual optical properties of frustules could be used in practical applications. Gale et al. [30] demonstrated the use of an antibodyfunctionalized diatom biosilica frustule as a microscale biosensor platform for the selective and label-free photoluminescence-based detection of biomolecules. Kong et al. [31] fabricated a photonic biosilica SERS substrate by integrating Ag NPs into microchannels of diatom frustules to identify explosive molecules in nanoliter solutions.

To the best of our knowledge, the present paper represents the first report of the application of a SERS immunoassay based on diatom biosilica to the detection of interleukin 8 (IL-8) in human blood plasma. IL-8 is an inflammatory cytokine that also plays an important role in breast cancer. There have been a few studies of the biological activity of this cytokine; for instance, Yokoe et al. [32] measured serum IL-8 in 12 heavily pretreated patients with recurrent breast cancer, and reported that IL-8 levels were higher in patients with refractory progressive disease but were almost unchanged in patients showing a partial response or no change after systemic therapy. In this paper we demonstrate the use of diatom biosilica as a SERS immune substrate.

\section{Methods}

\section{Reagents}

Recombinant human interleukin 8 (CXCL8) and monoclonal anti-interleukin- 8 antibody produced in mouse (clone 6217) were purchased from Sigma (St. Louis, MO, USA) and used as received. 5,5'-Dithiobis(2-nitrobenzoic acid) (DTNB), Lascorbic acid, gold(III) chloride trihydrate, trisodium citrate dihydrate, hexadecyltrimethylammonium bromide (CTAB), aminopropyltriethoxylsilane (APTES), bovine blood plasma albumin (BSA), and phosphate-buffered saline (PBS) packs (10 mM, pH 7.2) were also obtained from Sigma. 


\section{Blood sample preparation}

In our experiments, we used human blood samples from 10 healthy volunteers. These samples were made available courtesy of the Regional Blood Center (Warsaw, Poland). The samples underwent morphological analyses prior to use and revealed no abnormalities. All experiments were performed in compliance with the relevant laws and institutional guidelines. The study protocol was approved by the Ethics and Bioethics Committee of Cardinal Stefan Wyszynski University in Warsaw.

\section{Fabrication of diatom biosilica substrates}

A culture of the diatom species (Pseudostaurosira trainorii) was cultivated using Erlenmeyer flasks with $\mathrm{f} / 2$ nutrient solution containing silica at a concentration of $7 \mathrm{mg} \mathrm{mL}^{-1}$ under aeration and a $12 \mathrm{~h}$ light/12 $\mathrm{h}$ darkness regime. The light was provided by two fluorescent lamps with an intensity of $1500 \mathrm{~lx}$. The setup used for algae cultivation and a light microscopy image of living diatoms in the form of long colonial chains are shown in Fig. 1. After they had grown, the diatoms were washed out and treated with hydrogen peroxide in order to isolate silica frustules from the organic cellular matter according to the procedure described by Yang et al. [33]. The production capacity of the diatom species was about $320 \mathrm{mg}$ (dry weight) per liter.

\section{Fabrication of the immune platform}

Diatom frustules are mainly composed of amorphous hydrated $\mathrm{SiO}_{2}$ and are thus amenable to simple chemical functionalization for multiple applications (e.g., in photonics and analytical chemistry). To make the diatom frustules

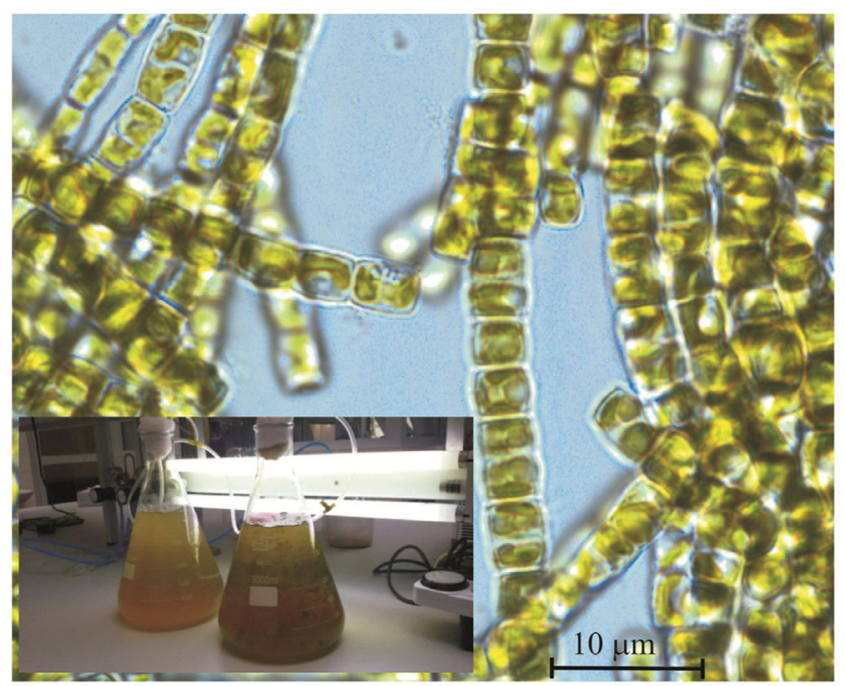

Fig. 1 Images of the algae cultivation setup and colonial chains of living diatoms (biosilica) useful for immune biosensing, glass slides were modified with the frustules, which was achieved as follows. The biosilica was dispersed in ethanol at a concentration of $0.5 \mathrm{mg} \mathrm{mL}^{-1}$. The cleaned glass slides were covered with $100,300,800$, and $1000 \mu \mathrm{l}$ of this diatom biosilica solution, respectively. All the samples were placed in an oven for $1 \mathrm{~h}$ at $450{ }^{\circ} \mathrm{C}$. In the next step, the surface silanol groups of the biosilica were functionalized with amine groups using aminopropyltriethoxylsilane (APTES) according to the procedure described by Yang et al. [33]. The glass slides with diatom frustules were incubated in $3 \mathrm{~mL}$ of a $1 \%$ solution of APTES in ethanol for $1 \mathrm{~h}$ at $50{ }^{\circ} \mathrm{C}$. The substrates were then removed from solution and rinsed with ethanol and DI water after $5 \mathrm{~min}$. The amino-modified surfaces were functionalizedd with anti-interleukin-8 (anti-IL-8) antibodies by immersing the frustules in a mixture of $25 \mu \mathrm{L}$ of $150 \mu \mathrm{g} \mathrm{mL}^{-1}$ anti-IL-8 in phosphate-buffered saline (PBS) and $5 \mu \mathrm{L}$ of activation solution (0.2 M EDC/0.05 M NHS; mixture in deionized water). Covalent bonds were formed between activated carboxyl groups on the anti-IL-8 antibodies and free amino groups on the diatoms. After $1 \mathrm{~h}$, the substrates with immobilized antibodies were rinsed with DI water and dried with nitrogen gas. Remaining active surface groups were blocked by immersing the substrates in $15 \mu \mathrm{L}$ of $2 \%$ BSA in PBS buffer solution (pH 7.2). Finally, the samples were rinsed twice with $5 \mathrm{~mL}$ of $10 \mathrm{mM}$ PBS buffer solution and stored at $4{ }^{\circ} \mathrm{C}$ in PBS for future use.

\section{Synthesis of Au nanoparticles}

Gold nanoparticles $70 \mathrm{~nm}$ in diameter (see Fig. S1 in the "Electronic supplementary material," ESM) and capped with CTAB (AuNPs@CTAB) were obtained via a two-step seeding protocol [34]. In the first step, AuNPs $15 \mathrm{~nm}$ in diameter were synthesized using the Turkevich approach [35]. In this approach, seeds were obtained by first adding $\mathrm{HAuCl}_{4} .3 \mathrm{H}_{2} \mathrm{O}(49 \mathrm{mg}, 0.125 \mathrm{mmol})$ to $250 \mathrm{~mL}$ of boiling water and then adding trisodium citrate dihydrate $(125 \mathrm{mg}$, $0.425 \mathrm{mmol})$. In a second step, CTAB $(2.74 \mathrm{~g}, 7.5 \mathrm{mmol})$ was dissolved in $500 \mathrm{~mL}$ of water heated to $35^{\circ} \mathrm{C}$. A solution of $\mathrm{HAuCl}_{4} .3 \mathrm{H}_{2} \mathrm{O}$ in water $(2.5 \mathrm{~mL}, 0.1 \mathrm{M})$ was then added and stirring was continued until the mixture became clear. Next, a solution of ascorbic acid $(2 \mathrm{~mL}, 2.5 \mathrm{mM})$ was injected and, after complete discoloration of the reaction mixture, $6.5 \mathrm{~mL}$ of the seed solution were added quickly. The mixture was stirred gently for $1 \mathrm{~h}$ and then centrifuged and carefully decanted. The precipitate was dispersed in $8 \mathrm{~mL}$ of CTAB water solution $(0.1 \mathrm{M})$ and left overnight to allow the shapeselective separation of nonspherical from spherical particles formed during the growing process. The solution of spherical AuNPs@CTAB was then carefully collected from above the sediment of unwanted nonspherical particles. The 
concentration of gold atoms in the final solution, as determined from the absorption spectrum, was $15.42 \mathrm{mM}$.

\section{Synthesis of anti-IL18/AuNPs-DTNB}

The solution of AuNPs@CTAB (1 mL)was centrifuged (6000 rpm, $10 \mathrm{~min}$ ) in order to remove excess CTAB. The supernatant was carefully decanted and the precipitate was dissolved in water $(1 \mathrm{~mL})$. A solution of DTNB $(18 \mathrm{mg}$, $0.045 \mathrm{mmol}$, in $5 \mathrm{~mL}$ of ethanol) was added during stirring and the obtained mixture was left overnight. The AuNPs@DTNB were collected by centrifugation (2000 rpm, $10 \mathrm{~min}$ ) and purified by sixfold dissolution in acetonitrile, precipitation with methanol, and centrifugation $(6000 \mathrm{rpm}$, $10 \mathrm{~min}$ ) and then by tenfold dissolution in water and centrifugation (7500 rpm, $10 \mathrm{~min}$ ). The purified AuNPs were dried and suspended in $2.5 \mathrm{~mL}$ of water. Next, the $10 \mu \mathrm{L}$ of solution containing the DTNB-modified Au nanoparticles were mixed with the solution of anti-IL8 in PBS buffer $(5 \mu \mathrm{L}$, $\left.60 \mu \mathrm{g} \mathrm{mL}^{-1}\right)$. For the synthesis of conjugate, the coupling

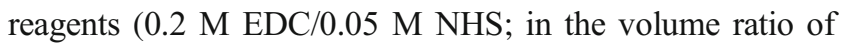
$5: 1$, in deionized water) were added and then the resulting mixture was incubated at $4{ }^{\circ} \mathrm{C}$ for 4 hours. The Ramanreporter-labeled immune Au nanoparticles (anti-IL8/AuNPsDTNB) were separated from the solution by centrifugation at $10,000 \mathrm{rpm}$ for $5 \mathrm{~min}$. The suspensions of anti-IL8/AuNPsDTNB were then passivated with $2.5 \mu \mathrm{L}$ of $2 \%$ BSA in PBS buffer solution. After $2 \mathrm{~h}$, the mixture was centrifuged again for $10 \mathrm{~min}$ at 20,000 rpm and then re-suspended in $1 \mathrm{~mL}$ of the PBS solution. The prepared anti-IL8/AuNPs-DTNB were stored at $4{ }^{\circ} \mathrm{C}$ for future use. The maximum number of antibodies bound to a nanoparticle was estimated to be ca. 540 [36]. The average diameter of a gold nanoparticle was about $70 \mathrm{~nm}$ according to SEM images and a histogram of Au nanoparticle diameters (ESM Figs. S1 and S2).

\section{Immunoassay protocol}

Immune reactions between antigens and antibodies were performed in a sandwich-type SERS immunoassay, as schematically illustrated in Fig. 2.

The first layer of this sandwich structure is composed of immobilized antibodies against IL- 8 captured on the amino-modified biosilica substrate. The second layer contains the complementary interleukins (proteins) captured by these selective antibodies. The third layer consists of Raman reporter DTNB-labeled immune Au nanoparticles. The presence of IL- 8 in the analyzed samples is identified through the appearance of the SERS spectrum of the DTNB bonded with the specific antibody against the studied interleukin.
Washing conditions

To limit nonspecific binding interactions, the immunoassay was washed three times with PBS buffer solution ( $\mathrm{pH}$ 7.2).

\section{Instrumentation}

\section{Raman and SERS measurements}

SERS measurements were performed using a Renishaw (Wotton-under-Edge, UK) inVia Raman system equipped with a $632-\mathrm{nm}$ He-Ne laser excitation source. The light from the laser was passed through a line filter and focused on a sample mounted on an $x-y-z$ translation stage with a $50 \times$ microscope objective (N.A. $=0.75$ ). The Raman-scattered light was collected by the same objective through a holographic notch filter to block out Rayleigh scattering. A grating with 1800 grooves per mm was used to provide a spectral resolution of $5 \mathrm{~cm}^{-1}$. The Raman scattering signal was recorded by a $1024 \times 256$ pixel RenCam CCD detector. The beam diameter was approximately $5 \mu \mathrm{m}$. Typically, the SERS spectra were recorded over an integration time of $30 \mathrm{~s}$ with a laser output power of $2.5 \mathrm{~mW}$ by mapping an area of size $50 \mu \mathrm{m} \times 50 \mu \mathrm{m}$.

\section{Electron microscopy characterization}

The morphological and structural features of the cleaned diatom frustules were examined by high-resolution scanning electron microscopy (SEM). SEM measurements of diatoms on the glass slide were done under high vacuum using the FEI (Hillsboro, OR, USA) Nova NanoSEM 450 with an accelerating voltage of between 2 and $30 \mathrm{kV}$. The glass slide was attached to the SEM table with conductive silver paste.

The elemental composition of the diatom frustules was determined using transmission electron microscopy (FEI Tecnai F20 X-Twintool) coupled with energy-dispersive Xray detection (EDX), with the sample being placed on a carbon-coated copper grid (lacey carbon support film, 400 mesh). In the study, samples of diatom frustules immobilized on glass slides and diatom frustules dispersed in ethanol were used.

\section{Results and discussion}

\section{Characterization of the substrate and Raman-reporter-labeled immune Au nanoparticles}

According to research results obtained by Sprynskyy et al. [37], the examined biosilica can be characterized as a macroporous material that contains a network of meso- and micropores with a BET surface area of $16.9 \mathrm{~m}^{2} / \mathrm{g}$. The pores 
b

$\mathbf{a}$

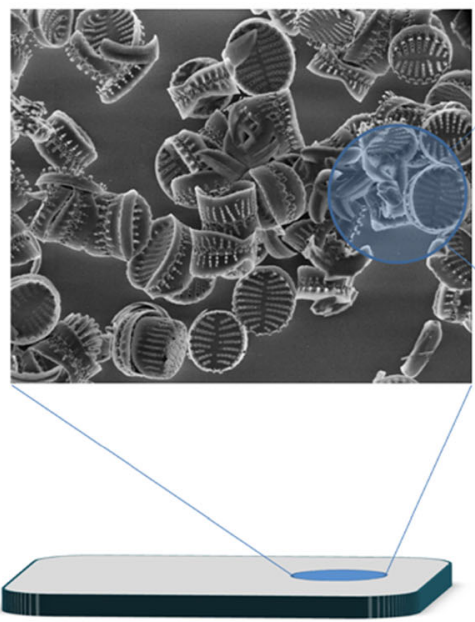

Fig. 2 a-d Schematic illustration of the SERS-based immunoassay: a glass slide was modified with diatom frustules (b shows an SEM image of a portion of the modified slide); $\mathbf{c}$ antibody capture and immobilization

showed size ranges of 1-1.5 nm, 10-20 nm, and 25-70 nm. The biosilica was identified as opal-A by X-ray diffraction and was termed "naturally organic functionalized 3D silica" due to the presence of residual organic functional groups related to biosilica-associated proteins as well as the functional groups characteristic of the amorphous silica framework. Also, the biosilica exhibited photoluminescence in the mid-ultraviolet region as well as in the blue-green region of the visible spectrum [37].

Scanning electron microscopy images of the diatom frustules used in our studies, which were isolated from cultivated diatoms of Pseudostaurosira trainorii, are presented in Fig. 3.

Figure 3 a shows an assembly of uniform and wellpreserved diatom frustules. The external and internal surfaces as well as sidewall regions of the diatom valves can also be observed. It is clear that the valves possess round to slightly elliptical forms with an average diameter of 4$5 \mu \mathrm{m}$. The inset in Fig. 3a shows a macroscopic image of the dried diatom frustules. Figure $3 b$ reveals the intricate

\section{d}

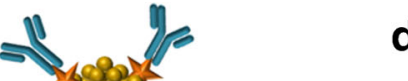

d 
$(\mathrm{Zn}, \mathrm{Cu}, \mathrm{Co})$ in the nutrient solution were an order of magnitude lower still.

In order to optimize the performance of the SERS-based immunoassay for sensing IL-8, immune platforms with different densities of diatom frustules on the surfaces of the glass slides were trialled. Figures $4 \mathrm{a}-\mathrm{d}$ are SEM images of slides with $100,300,800$, and $1000 \mu \mathrm{L}$ of the diatom frustule solution $(0.5 \mathrm{mg}$ diatoms per $\mathrm{mL}$ of ethanol), respectively, which show how the glass slides differ in frustule coverage.

It is clearly apparent that very dense frustule packing was obtained by drop-casting multiple coats of the $800 \mu \mathrm{L}$ of $0.5 \mathrm{mg} \mathrm{mL}^{-1}$ frustule solution onto the glass substrate.

Subsequently, the SERS activities of the DTNB-labeled immune Au nanoparticles were examined. The successful binding of the DTNB and IL-8 antibodies to the Au nanoparticles was demonstrated by UV experiments (ESM Fig. S4). As shown in Fig. S4a in the ESM, the as-received gold nanoparticle solution has a strong extinction maximum at $609 \mathrm{~nm}$. This wavelength is indicative that the individual nanoparticles average approximately $70 \mathrm{~nm}$ in diameter. The spectrum of AuNPs redshifted a little after coating them with the Raman reporter (ESM Fig. S4b), as the LSPR band of AuNPs is very sensitive to the refractive index of the surrounding medium. The redshift of the surface plasmon resonance peak [38] demonstrates the successful binding of DTNB to the gold nanoparticles. After modification with IL-8 antibodies, there was a large decrease in the strength of this band and it redshifted from $609 \mathrm{~nm}$ to $637 \mathrm{~nm}$, which indicates that residual surface vacancies of DTNB-labeled AuNPs are occupied by anti-IL-8 antibodies (ESM Fig. S4c). Similar results have been observed and detected using the ATR/FTIR technique [39]. Additionally, according to the literature [40], the broadening and redshift may also indicate aggregation.

Figure $4 \mathrm{a}$ shows the SERS spectrum of DTNB chemisorbed onto AuNPs, which is very intense and dominated by bands at $1326 \mathrm{~cm}^{-1}$ and $1556 \mathrm{~cm}^{-1}$ arising from a symmetric stretching mode of nitro groups $\nu_{\mathrm{s}}\left(\mathrm{NO}_{2}\right)$ and an aromatic ring stretching mode, respectively [41]. The peaks at 1176 and $1054 \mathrm{~cm}^{-1}$ were attributed to $\mathrm{CH}_{3}$ rocking, $\mathrm{C}-\mathrm{N}$ stretching, and C-N bending [42]. Similarly, a strong characteristic SERS signal from DTNB (Fig. 5b) was obtained for the DTNB-labeled immune Au nanoparticles (after the antiIL-8 antibody immobilization), indicating that DTNB acts as a sensitive Raman reporter for SERS immune sensing.

\section{SERS immunoassay detection of IL-8}

In a first step, the four substrates with different frustule coverages (see SEM images in Fig. 4) were evaluated for their immune sensitivities according to the immunoassay protocol outlined above. All capturing surfaces were coated with anti-IL-8 antibodies and then exposed to a preselected concentration of antigen
Fig. 4 a-d SEM images of glass slides covered with a $100 \mu \mathrm{L}$, b $300 \mu \mathrm{L}, \mathbf{c} 800 \mu \mathrm{L}$, and $\mathbf{d} 1000 \mu \mathrm{L}$ of a $0.5 \mathrm{mg} \mathrm{mL}^{-1}$ diatom frustule solution
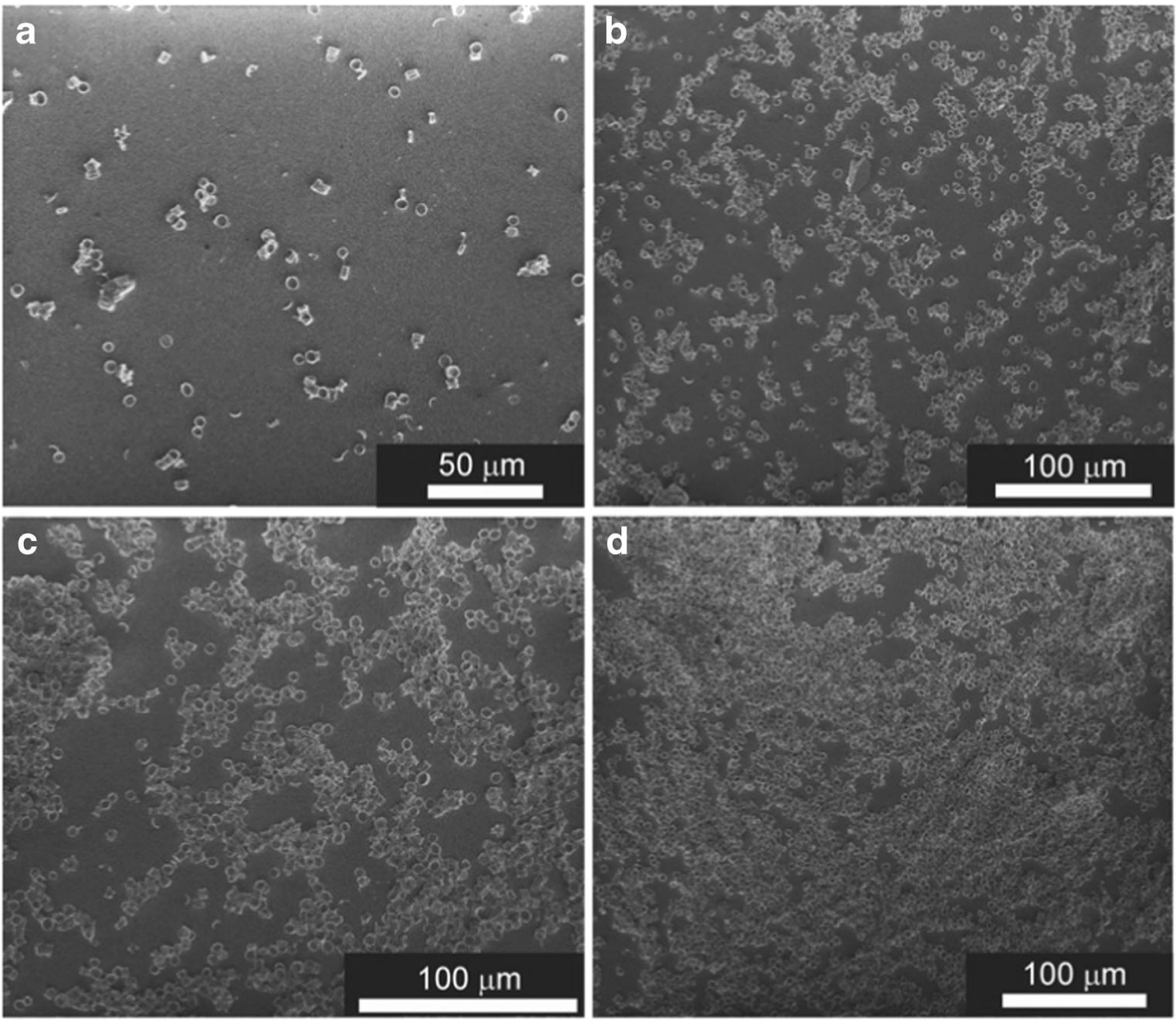


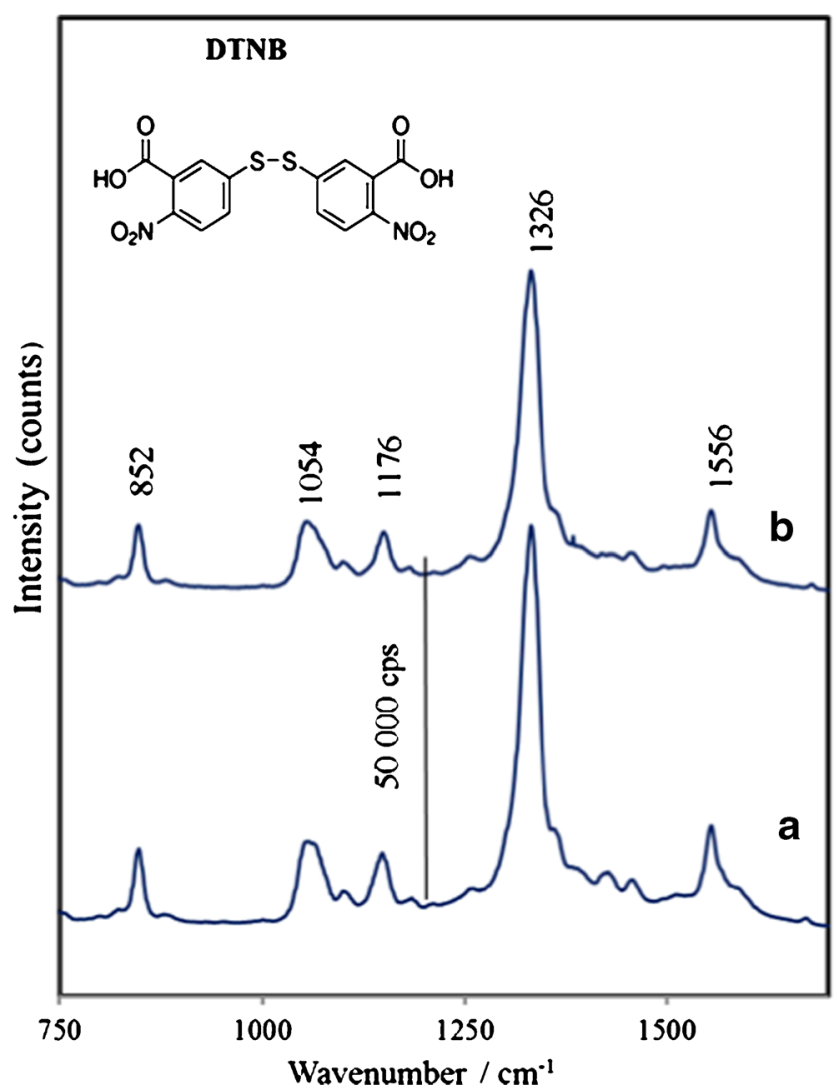

Fig. 5 a SERS spectrum of DTNB (Raman reporter) adsorbed onto AuNPs, and b SERS spectrum of DTNB-labeled immune-AuNPs

(25 $\mathrm{ng} \mathrm{mL}^{-1}$ in blood plasma). The SEM images in Fig. S6 (see the ESM) demonstrate the presence of DTNB-labeled immune $\mathrm{Au}$ nanoparticles on the diatom frustules on the substrates. Figure 6 shows how the intensity of the most prominent band (the marker band) of DTNB at $1326 \mathrm{~cm}^{-1}$ changes with the coverage of the capturing substrate by the diatom biosilica. As the biosilica density increases on the surface of the glass, the SERS signal of the marker band becomes stronger (Fig. 6a-c). The red line in the inset of Fig. 6 shows the critical density of diatoms on the surface. The SERS signal in Fig. 6d does not show any further increase due to the formation of the multilayer of diatoms and aggregates, which is not as effective at immunosensing (see ESM Fig. S5 for a SEM picture of the SERS platform obtained using $1 \mathrm{~mL}$ of the solution of diatoms). We found that this multilayer structure of diatom-based immune substrate exhibited a significant decrease in physical/chemical stability. To examine the strength of the immobilization of the diatoms on the glass, a Scotch tape test was performed. The morphologies of the two types of capturing substrates corresponding to monolayer and multilayer arrangements of diatom frustules (see Fig. 4c and d) were observed using SEM before and after placing Scotch tape on the substrates and then peeling them off. The multilayered immune substrate was removed almost completely from the surface of glass, while the monolayer of diatom frustules was only slightly affected by the tape. These

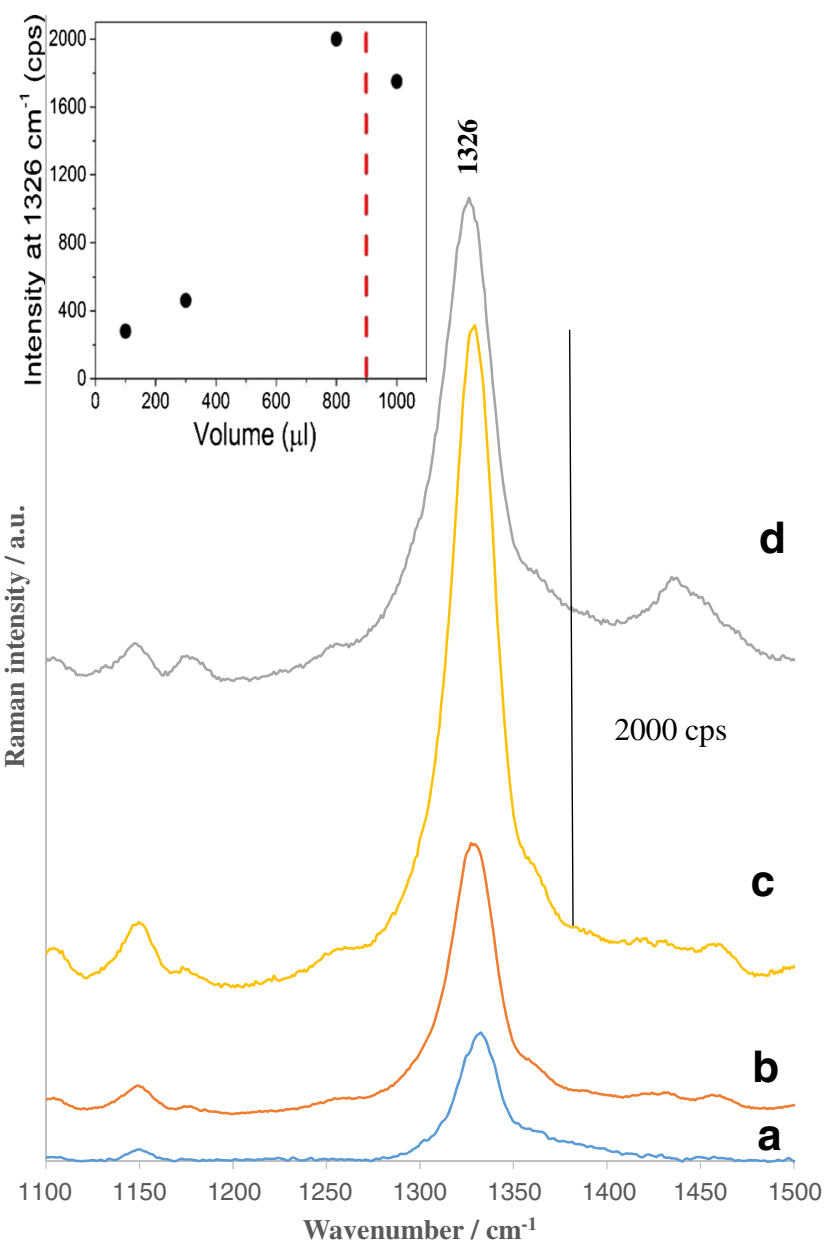

Fig. 6 a-d SERS spectra of the marker band at $1326 \mathrm{~cm}^{-1}$ with different densities of diatom frustules on the surface of the glass slide: $\mathbf{a} 100 \mu \mathrm{L}, \mathbf{b}$ $300 \mu \mathrm{L}, \mathbf{c} 800 \mu \mathrm{L}$, and $\mathbf{d} 1000 \mu \mathrm{L}$ of a $0.5 \mathrm{mg}$ diatoms per $\mathrm{mL}$ of ethanol solution. The inset presents the relationship between the intensity of the marker band at $1326 \mathrm{~cm}^{-1}$ and the density of diatom frustules on the surface of the glass slide

experiments indicated that the morphology and the strength of bonding of the diatom frustules with the glass substrate are important influences on the stability and sensitivity of the immunosensor.

These experimental results prove that the morphology of the capturing substrate presented in Fig. $4 \mathrm{c}$ is best suited to achieving the highest IL-8 detection sensitivity, and so this substrate was applied to a quantitative analysis of IL-8 in plasma blood samples.

\section{Quantitative analysis}

The applicability of the developed SERS-based immunoassay to the quantitative analysis of IL- 8 was evaluated by measuring the SERS response for varying concentrations of the target IL-8 in blood plasma samples. A dilution series of IL-8 in human blood plasma covering the range $0-30 \mathrm{ng} \mathrm{mL}^{-1}$ was prepared. This concentration range was chosen to cover 


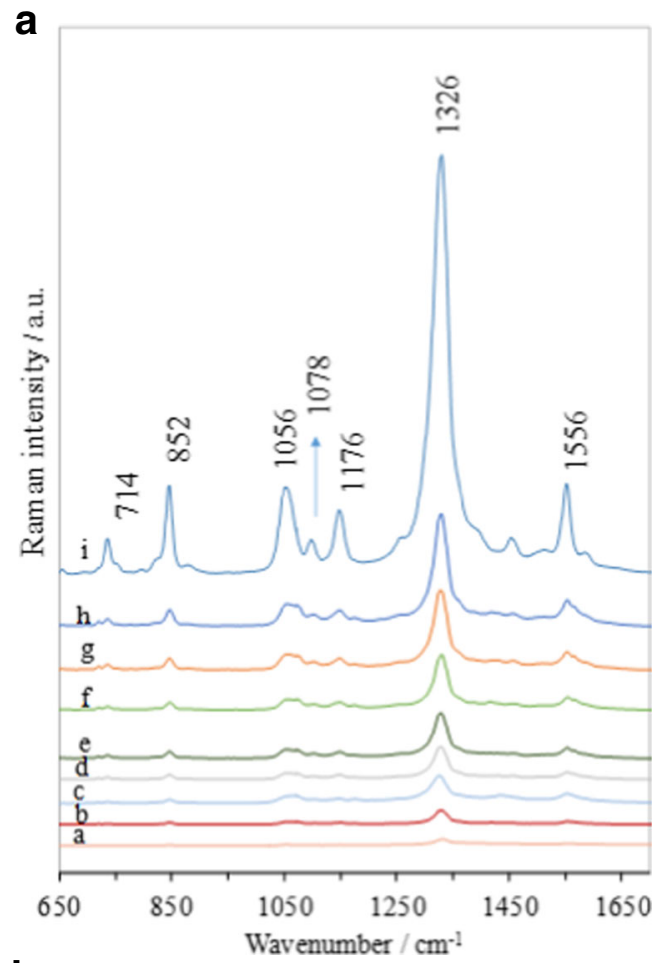

b

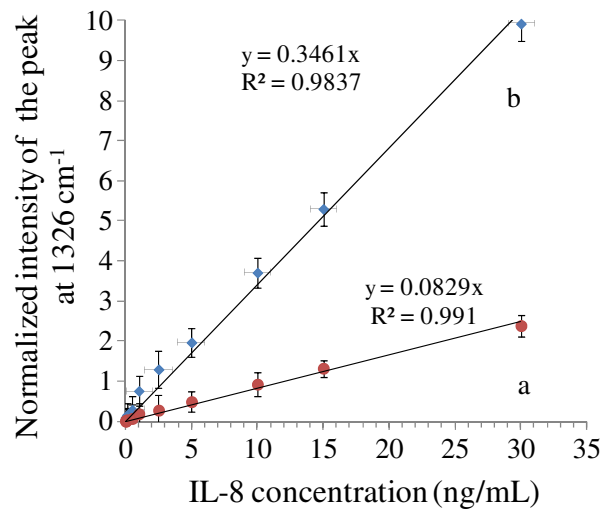

Fig. 7 (A) SERS spectra obtained for various concentrations of IL-8: (a) 0.0 ; (b) $0.005 ;$ (c) $0.01 ;$ (d) $0.1 ;$ (e) $0.5 ;$ (f) $1.0 ;$ (g) $2.5 ;$ (h) $5.0 ;$ (i) 30.0 ng $\mathrm{mL}^{-1}$ in blood plasma. (B) Relationship between the normalized intensity of the marker band at $1326 \mathrm{~cm}^{-1}$ and the concentration of IL-8 in the range from 0 to $30 \mathrm{ng} \mathrm{mL}^{-1}$ for (a) a glass slide with no diatom frustules and (b) a glass slide with diatom frustules deposited on its surface. Each SERS spectrum is the average of 20 measurements obtained at different points on the SERS substrate surface using the mapping mode. Each error bar indicates the standard deviation calculated from 20 measurements obtained at different spots on the surface for a particular IL-8 concentration

clinically relevant IL-8 concentrations. Figure 7a presents SERS spectra obtained for selected concentrations of IL-8 after completing the immunoassay protocol described earlier in this paper. The intensity of the average SERS fingerprint spectrum of the Raman reporter (DTNB) increased linearly with the concentration of the target IL- 8 in the sample over the whole concentration range tested. Note that, in order to achieve reproducible data, the confocal Raman mapping mode was employed to solve any problems with a nonuniform spatial distribution of SERS nanoparticles (aggregations) on the SERS substrate. A blank spectrum (Fig. 7a) was recorded by analyzing a blood plasma sample from a healthy subject without any added IL- 8 antigen. When the complementary antigen is not added, the Raman-reporter-labeled immune Au nanoparticles should be easily removed from the substrate by washing. The presence of a weak peak at $1326 \mathrm{~cm}^{-1}$ highlights the high sensitivity of this assay, as IL- 8 is present in normal healthy blood at picoogram concentrations [32]. Calibration curve (a) in Fig. 7b was obtained by plotting the normalized intensity of the SERS signal of the DTNB marker band at $1326 \mathrm{~cm}^{-1}$ against the concentration of the antigen in the range from 0 to $30 \mathrm{ng} \mathrm{mL}^{-1}$. Each error bar indicates the standard deviation determined from ten measurements at different spots for a particular concentration. In the linear region, the best-fit equation for the calibration curve was $y=0.3462 x$ and the coefficient of determination $\left(R^{2}\right)$ was 0.98 . For the linear calibration curve, it was assumed that the SERS intensity at $1326 \mathrm{~cm}^{-1}(y)$ is linearly related to the concentration of the target interleukin $(x)$. In addition, the low detection limit (LOD) was estimated using the signal-to-noise $(\mathrm{S} / \mathrm{N})$ method [43]. A S/N ratio of three is generally employed to estimate the LOD. Based on these data, the low detection limit was calculated to be $6.2 \mathrm{pg} \mathrm{mL}^{-1}$.

In a first step, a control experiment was performed to verify the specificity of the immunological recognition. The unspecific Akt blocking peptide for anti-IL-8 antibody was employed in the same immunoassay protocol as described for Fig. 2. The Akt blocking peptide specifically binds only to the Akt (pan) Rabbit mAb antibody. As can be seen in Fig. S8a of the ESM, after the Akt blocking peptide was added, there were no strong bands, only two extremely weak bands at about $840 \mathrm{~cm}^{-1}$ and $1326 \mathrm{~cm}^{-1}$ originating from the Raman reporter molecules. This may indicate that a small number of DTNB-labeled immune AuNPs were adsorbed onto the capturing substrate without any immune recognition. However, more importantly, the presence of these marker bands demonstrates that this technique can detect the low levels of IL-8 that are usually present in normal control blood plasma samples. When the anti-IL8/AuNPs-DTNB were incubated with the IL-8 in the plasma samples, strong signals originating from the Raman reporter molecules appeared in the SERS spectrum (ESM Fig. S7b-d). As can be seen in Fig. S7 of the ESM, the difference in intensity between the SERS signals from DTNB that were obtained in the specific and unspecific recognition regimes clearly demonstrates the high specificity of this immunocomplex.

In our study, we also compared the efficiency of the developed SERS immunoassay with that of a conventional SERS sensor based on flat glass. The glass slide was employed in the same immunoassay protocol as described earlier in this paper. 


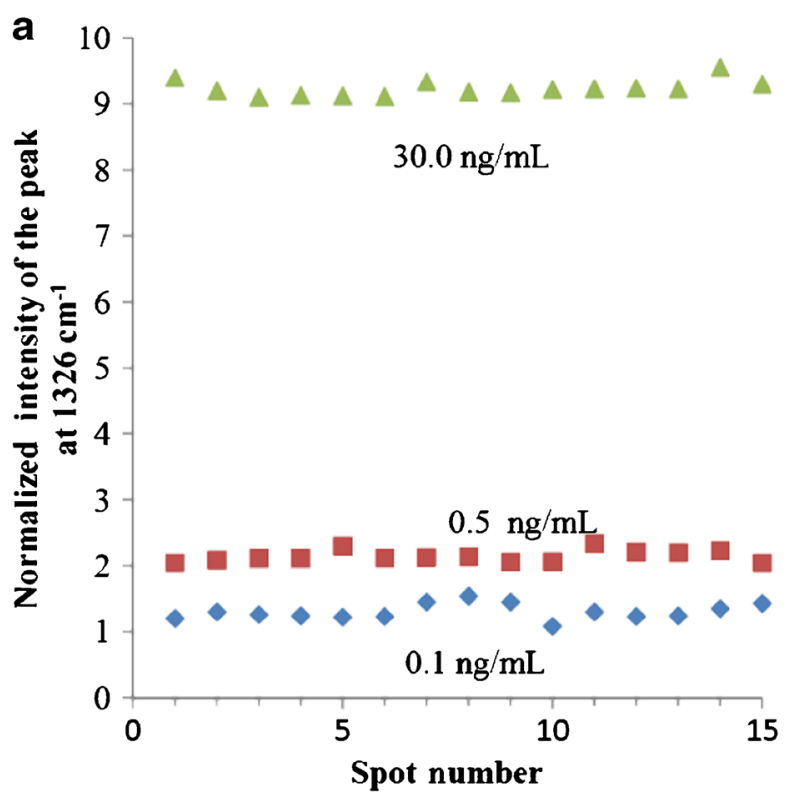

Fig. 8 a Reproducibility of three separately prepared SERS immunoassays exposed to different concentrations of IL-8 in blood plasma $\left(0.1,0.5\right.$, and $\left.30.0 \mathrm{ng} \mathrm{mL}^{-1}\right)$. SERS spectra were recorded at 15 randomly selected spots on the substrate in each SERS assay. b Representative two-dimensional SERS spectra recorded in SERS assays

Based on the constructed SERS response curve (b) shown in Fig. $7 \mathrm{~b}$, the lowest detectable concentration was $2.5 \mathrm{ng} \mathrm{mL}^{-1}$, which is two orders of magnitude worse than that obtained for the diatom-frustule-based SERS immune substrate. Although the reason for this enhanced sensitivity of the technique developed here requires further study as it is a rather complex phenomenon, it is very likely due to the unique properties of the diatom frustules.

Diatoms are photonic crystals that can increase the local field intensity, leading to Raman signal enhancement [44] and thus improving the detection limit of the SERSbased immunoassay sensor. The periodic two-dimensional pore arrays permit guided-mode resonances (GMRs) at visible wavelengths. Theoretical and experimental investigations indicate that the electric field amplitude of localized surface plasmons (LSPs) for nonmetallic structures can be significantly amplified through the coupling of the LSPs with the plasmonic structure of the diatom [45]. As a result, the presence of the diatom frustules lowers the detection limit for IL-8 to $6.2 \mathrm{pg} \mathrm{mL}^{-1}$, which is two orders of magnitude better than that achieved with a conventional glass-based SERS- immunoassay. Apart from the sensitivity, there are also other advantages of the SERS-based immunoassay sensor based on diatoms. The porous morphology of the diatom frustules leads to unique physical and chemical properties of the frustules, such as rapid mass transport inside the microchannels and outstandingly high surface area [46]. In the SERS-based assay reported here, this large surface area can magnify

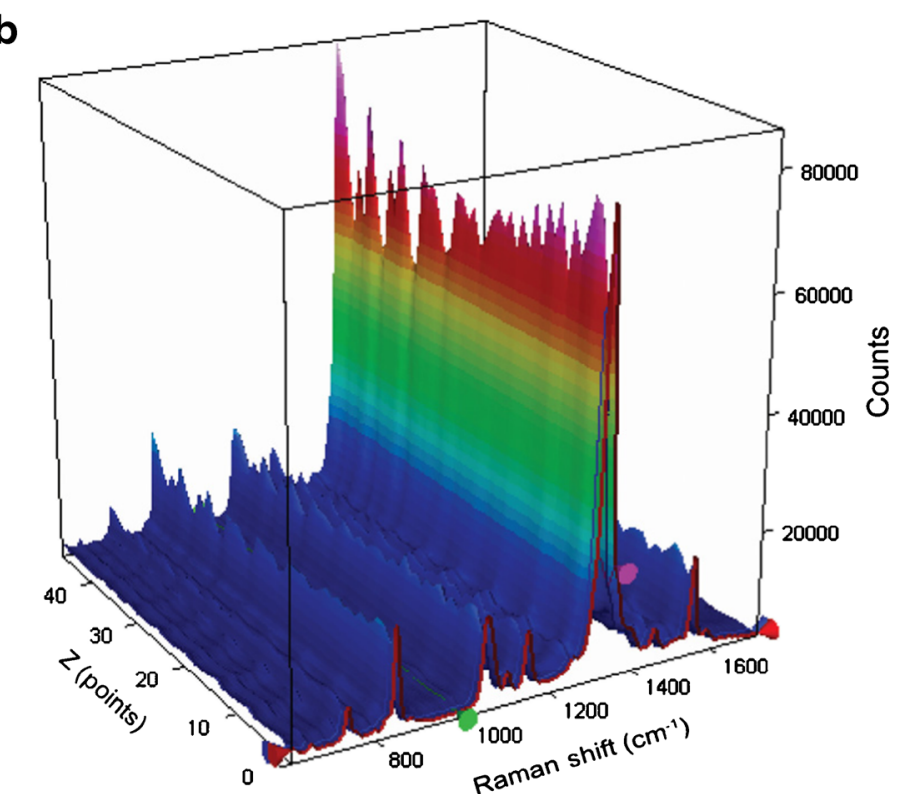

of $30.0 \mathrm{ng} \mathrm{mL} \mathrm{m}^{-1} \mathrm{IL}-8$ performed at 40 different spots on the SERS surface. The spectra were collected over a distance of $1 \mathrm{~mm}$ in $10 \mu \mathrm{m}$ steps (40 spectra are shown). Each point on the map was recorded using $5 \mathrm{~mW}$ of excitation at $632 \mathrm{~nm}$ and an integration time of $10 \mathrm{~s}$

the number of hotspots and adsorption sites for the analyte compared with planar glass or metallic nanostructures [47], thus improving the immunoassay sensing. The abundant hydroxyl groups on their surfaces make diatom frustules very hydrophilic in comparison with a flat glass slide. Their highly hydrophilic surfaces with highly ordered nanopores of diatoms can drive the liquid flow from the glass towards the diatom frustule as a result of capillary forces [48]. In effect, the highly hydrophilic and porous diatom frustules provide a driving force that concentrates the target molecules, which may lead to a reduction in the detection limit of several orders of magnitude [49].

The current detection limit for IL-8 using a conventional ELISA test is about $15.6 \mathrm{pg} \mathrm{mL}^{-1}$ [50], which does not always meet the requirements of clinical diagnosis. Our method improves on that detection limit and potentially permits the quantitative detection of interleukins in complex biological fluids.

The reproducibility of the presented SERS immunoassay for IL-8 detection was also investigated. Figure 8 shows 15 individual readings from 15 randomly selected spots on three different biosilica-based SERS surfaces that were immersed in human blood plasma samples with different concentrations of IL-8 $\left(0.1,0.5\right.$, and $\left.30.0 \mathrm{ng} \mathrm{mL}^{-1}\right)$. To get a statistically valid result, the marker band of the Raman reporter at $1326 \mathrm{~cm}^{-1}$ was utilized to calculate the relative standard deviation (RSD). The corresponding relative standard deviations were 9.5, 8.0, and 7.2\%, respectively. The relative average standard deviation (RSD) of 
this method was found to be less than $9 \%$, which is comparable to that of a conventional ELISA assay.

\section{Conclusions}

We have demonstrated, for the first time, a SERS-based immunoassay that utilizes naturally created photonic biosilica to detect the interleukin IL-8 in human plasma samples.

The most notable features of the developed SERS immunoassay include (i) the creation of an immune substrate based on anti-IL-8-functionalized diatom biosilica and (ii) the application of appropriately designed Raman-reporter-labeled AuNPs, which produce very strong SERS enhancement. The DTNB-labeled immune AuNPs can form a sandwich structure with the antigen and the antibody, as shown by the characteristic spectrum of the Raman reporter molecules (DTNB). The estimated lower detection limit and average standard deviation of the selected marker band for IL- 8 at $1326 \mathrm{~cm}^{-1}$ show that the method is highly sensitive to clinically relevant concentrations of this interleukin and has excellent reproducibility, which is desirable for analytical analysis. This SERS assay also exhibits high biological specificity for the detection of IL-8 in complex fluids. Furthermore, the experimental results confirm that diatom frustules amplify the sensitivity of the immunosensor in comparison to a conventional sensor based on flat glass. The detection limits for IL-8 on diatom frustules and when using the glass-based SERS immune substrate in human blood plasma were found to be $6.2 \mathrm{pg} \mathrm{mL}^{-1}$ and $2.5 \mathrm{ng} \mathrm{mL}^{-1}$.

The SERS immunoassay presented here can be used for the sensitive and selective detection of immune markers in biological fluids and for point-of-care analysis.

Acknowledgements Authors would like to acknowledge the support from the National Science Centre under grant UMO-2015/17/B/ST4/ 04128.

Compliance with ethical standards All experiments were performed in compliance with the relevant laws and institutional guidelines. The protocol of the study was approved by the Ethics and Bioethics Committee of Cardinal Stefan Wyszynski University in Warsaw. Informed consent was obtained from all patients.

Conflict of interest The authors declare no conflict of interest.

Open Access This article is distributed under the terms of the Creative Commons Attribution 4.0 International License (http:// creativecommons.org/licenses/by/4.0/), which permits unrestricted use, distribution, and reproduction in any medium, provided you give appropriate credit to the original author(s) and the source, provide a link to the Creative Commons license, and indicate if changes were made.

\section{References}

1. Brocker C, Thompson D, Matsumoto A, Nebert DW, Vasiliou V. Evolutionary divergence and functions of the human interleukin (IL) gene family. Hum Genomics. 2010;5(1):30-55.

2. Commins SP, Borish L, Steinke JW. Immunologic messenger molecules: cytokines, interferons, and chemokines. J Allergy Clin Immunol. 2010;125:S53-72.

3. Aziz N, Nishanian P, Fahey JL. Levels of cytokines and immune activation markers in plasma in human immunodeficiency virus infection: quality control procedures. Clin Diagn Lab Immunol. 1998;5(6):755-61.

4. Maciel R. Standard curve fitting in immunodiagnostics: a primer. J Clin Immunoass. 1985;8:98-106.

5. Mosmann TR, Fong TA. Specific assays for cytokine production by T cells. J Immunol Methods. 1989;116(2):151-8.

6. Naylor MS, Relf M, Balkwill F. Northern analysis, ribonuclease protection, and in situ analysis of cytokine messenger RNA. Cytokines: a practical approach. Oxford: Oxford University Press; 1995. p. 35-6.

7. Sander B, Hoiden I, Andersson U, Moller E, Abrams JS. Similar frequencies and kinetics of cytokine producing cells in murine peripheral blood and spleen. Cytokine detection by immunoassay and intracellular immunostaining. J Immunol Methods. 1993;166(2):201-14.

8. Carson RT, Vignali DA. Simultaneous quantitation of 15 cytokines using a multiplexed flow cytometric assay. J Immunol Methods. 1999;227(1-2):41-52.

9. Gearing A, Cartwright J, Wadhwa M. Biological and immunological assays for cytokines. In: Thomson A, editor. The cytokine handbook. London: Academic Press; 1991. p. 339-55.

10. Whicher J, Ingham E. Cytokine measurements in body fluids. Eur Cytokine Netw. 1990;1(4):239-43.

11. Mire-Sluis AR, Gaines-Das R, Thorpe R. Immunoassays for detecting cytokines: what are they really measuring? J Immunol Methods. 1995;186(2):157-60.

12. Cannon JG, Nerad JL, Poutsiaka DD, Dinarello CA. Measuring circulating cytokines. J Appl Physiol. 1993;75(4):1897-902.

13. Sharma B, Frontiera RR, Henry A-I, Ringe E, Van Duyne RP. SERS: materials, applications, and the future. Mater Today. 2012;15(1-2):16-25.

14. Campion A, Kambhampati P. Surface-enhanced Raman scattering. Chem Soc Rev. 1998;27(4):241-50.

15. Camden JP, Dieringer JA, Wang Y, Masiello DJ, Marks LD, Schatz $\mathrm{GC}$, et al. Probing the structure of single-molecule surface-enhanced Raman scattering hot spots. J Am Chem Soc. 2008;130(38):12616-7.

16. Stiles PL, Dieringer JA, Shah NC, Van Duyne RP. Surface-enhanced Raman spectroscopy. Annu Rev Anal Chem. 2008;1:601-26.

17. Le Ru EC, Etchegoin PG. Single-molecule surface-enhanced raman spectroscopy. Annu Rev Phys Chem. 2012;63:65-87.

18. El-Ansary A, Faddah LM. Nanoparticles as biochemical sensors. Nanotechnol Sci Appl. 2010;3:65-76.

19. Beier HT, Cowan CB, Chou IH, Pallikal J, Henry JE, Benford ME, et al. Application of surface-enhanced Raman spectroscopy for detection of beta amyloid using nanoshells. Plasmonics. 2007;2(2):55-64.

20. An J-H, El-Said WA, Yea C-H, Kim T-H, Choi J-W. Surfaceenhanced Raman scattering of dopamine on self-assembled gold nanoparticles. J Nanosci Nanotechno. 2011;11(5):4424-9.

21. Jun BH, Kim JH, Park H, Kim JS, Yu KN, Lee SM, et al. Surfaceenhanced Raman spectroscopic-encoded beads for multiplex immunoassay. J Comb Chem. 2007;9(2):237-44.

22. Wang Z, Zong S, Li W, Wang C, Xu S, Chen H, et al. SERSfluorescence joint spectral encoding using organic-metal-QD hybrid nanoparticles with a huge encoding capacity for high- 
throughput biodetection: putting theory into practice. J Am Chem Soc. 2012;134(6):2993-3000.

23. Wang Y, Salehi M, Schutz M, Schlucker S. Femtogram detection of cytokines in a direct dot-blot assay using SERS microspectroscopy and hydrophilically stabilized $\mathrm{Au}-\mathrm{Ag}$ nanoshells. Chem Commun. 2014;50(21):2711-4.

24. Wang Y, Salehi M, Schutz M, Rudi K, Schlucker S. Microspectroscopic SERS detection of interleukin-6 with rationally designed gold/silver nanoshells. Analyst. 2013;138(6):1764-71.

25. Kaminska A, Witkowska E, Winkler K, Dziecielewski I, Weyher JL, Waluk J. Detection of hepatitis B virus antigen from human blood: SERS immunoassay in a microfluidic system. Biosens Bioelectron. 2015;66:461-7.

26. Xu X, Hasan D, Wang L, Chakravarty S, Chen RT, Fan DL, et al. Guided-mode-resonance-coupled plasmonic-active $\mathrm{SiO}_{2}$ nanotubes for surface enhanced Raman spectroscopy. Appl Phys Lett. 2012;100(19): 191114.

27. De Stefano M, De Stefano L. Nanostructures in diatom frustules: functional morphology of valvocopulae in cocconeidacean monoraphid taxa. J Nanosci Nanotechno. 2005;5(1):15-24.

28. Ferrara MA, Dardano P, De Stefano L, Rea I, Coppola G, Rendina I, et al. Optical properties of diatom nanostructured biosilica in Arachnoidiscus sp.: micro-optics from Mother Nature. PLoS One. 2014;9(7):e103750.

29. Bismuto A, Setaro A, Maddalena P, De Stefano L, De Stefano M. Marine diatoms as optical chemical sensors: a time-resolved study. Sens Actuator B Chem. 2008;130(1):396-9.

30. Gale DK, Gutu T, Jiao J, Chang CH, Rorrer GL. Photoluminescence detection of biomolecules by antibodyfunctionalized diatom biosilica. Adv Funct Mater. 2009;19(6): 926-33.

31. Kong XM, Xi YT, Le Duff P, Chong XY, Li EW, Ren FH, et al. Detecting explosive molecules from nanoliter solution: a new paradigm of SERS sensing on hydrophilic photonic crystal biosilica. Biosens Bioelectron. 2017;88:63-70.

32. Yokoe T, Iino Y, Morishita Y. Trends of IL-6 and IL-8 levels in patients with recurrent breast cancer: preliminary report. Breast Cancer (Tokyo, Japan). 2000;7(3):187-90.

33. Yang J, Zhen L, Ren F, Campbell J, Rorrer GL, Wang AX. Ultrasensitive immunoassay biosensors using hybrid plasmonic-biosilica nanostructured materials. J Biophotonics. 2015;8(8):659-67.

34. Fernandez-Lopez C, Mateo-Mateo C, Alvarez-Puebla RA, PerezJuste J, Pastoriza-Santos I, Liz-Marzan LM. Highly controlled silica coating of PEG-capped metal nanoparticles and preparation of SERS-encoded particles. Langmuir. 2009;25(24):13894-9.

35. Enustun BV, Turkevich J. Coagulation of colloidal gold. J Am Chem Soc. 1963;85(21):3317-28.
36. Han XX, Kitahama Y, Itoh T, Wang CX, Zhao B, Ozaki Y. Proteinmediated sandwich strategy for surface-enhanced Raman scattering: application to versatile protein detection. Anal Chem. 2009;81(9):3350-5.

37. Sprynskyy M, Pomastowski P, Hornowska M, Król A, Rafińska K, Buszewski B. Naturally organic functionalized 3D biosilica from diatom microalgae. Mater Des. 2017;132:22-9.

38. Mie G. Beiträge zur Optik trüber Medien, speziell kolloidaler Metallösungen. Ann Phys. 1908;330(3):377-445.

39. Song CY, Wang ZY, Yang J, Zhang RH, Cui YP. Effects of solid substrate on the SERS-based immunoassay: a comparative study. J Raman Spectrosc. 2011;42(3):313-8.

40. Ni J, Lipert RJ, Dawson GB, Porter MD. Immunoassay readout method using extrinsic Raman labels adsorbed on immunogold colloids. Anal Chem. 1999;71(21):4903-8.

41. Guven B, Basaran-Akgul N, Temur E, Tamer U, Boyaci IH. SERSbased sandwich immunoassay using antibody coated magnetic nanoparticles for Escherichia coli enumeration. Analyst. 2011;136(4):740-8.

42. Grubisha DS, Lipert RJ, Park HY, Driskell J, Porter MD. Femtomolar detection of prostate-specific antigen: an immunoassay based on surface-enhanced Raman scattering and immunogold labels. Anal Chem. 2003;75(21):5936-43.

43. Shrivastava A, Gupta V. Methods for the determination of limit of detection and limit of quantitation of the analytical methods. Chron Young Sci. 2011;2(1):21-5.

44. Fuhrmann T, Landwehr S, El Rharbi-Kucki M, Sumper M. Diatoms as living photonic crystals. Appl Phys B. 2004;78(3-4):257-60.

45. Ren FH, Campbell J, Wang XY, Rorrer GL, Wang AX. Enhancing surface plasmon resonances of metallic nanoparticles by diatom biosilica. Opt Express. 2013;21(13):15308-13.

46. Deng Y, Qi D, Deng C, Zhang X, Zhao D. Superparamagnetic highmagnetization microspheres with an $\mathrm{Fe}_{3} \mathrm{O}_{4} @ \mathrm{SiO}_{2}$ core and perpendicularly aligned mesoporous $\mathrm{SiO}_{2}$ shell for removal of microcystins. J Am Chem Soc. 2008;130(1):28-9.

47. Ko H, Singamaneni S, Tsukruk VV. Nanostructured surfaces and assemblies as SERS media. Small. 2008;4(10):1576-99.

48. Buffone $\mathrm{C}$, Sefiane K. Investigation of thermocapillary convective patterns and their role in the enhancement of evaporation from pores. Int J Multiphas Flow. 2004;30(9):1071-91.

49. De Angelis F, Gentile F, Mecarini F, Das G, Moretti M, Candeloro $\mathrm{P}$, et al. Breaking the diffusion limit with super-hydrophobic delivery of molecules to plasmonic nanofocusing SERS structures. Nat Photonics. 2011;5(11):682-7.

50. DeForge LE, Remick DG. Sandwich ELISA for detection of picogram quantities of interleukin-8. Immunol Investig. 1991;20(1):89-97. 